\title{
Las riberas de los ríos mediterráneos y su calidad: el uso del índice QBR.
}

\author{
$\mathrm{M}^{\mathrm{a}}$ Luisa Suárez ${ }^{1}, \mathrm{M}^{\mathrm{a}}$ Rosario Vidal-Abarca ${ }^{1}, \mathrm{M}^{\mathrm{a}}$ del Mar Sánchez-Montoya ${ }^{1}$, \\ Javier Alba-Tercedor ${ }^{2}$, Maruxa Álvarez ${ }^{3}$, Juan Avilés ${ }^{4}$, Núria Bonada ${ }^{5}$, Jesús Casas ${ }^{6}$, \\ Pablo Jáimez-Cuéllar ${ }^{2}$, Antoni Munné 5 , Isabel Pardo ${ }^{3}$, Narcís Prat ${ }^{5}$, Maria Rieradevall ${ }^{5}$, \\ $\mathrm{M}^{\mathrm{a}}$ Jacoba Salinas ${ }^{7}$, Manuel Toro ${ }^{4}$ y Soledad Vivas ${ }^{6}$
}

${ }^{1}$ Departamento de Ecología e Hidrología. Universidad de Murcia. Campus de Espinardo. 30100 Murcia.

${ }^{2}$ Departamento de Biología Animal y Ecología. Universidad de Granada. Campus Universitario de Fuentenueva. 18071 Granada.

${ }^{3}$ Área de Ecología. Universidad de Vigo. Campus Lagoas-Marcosende. 36200 Vigo.

${ }^{4}$ CEDEX. División de Ecología de los Sistemas Acuáticos Continentales. Paseo Bajo Virgen del Puerto, 3. 28005 Madrid.

${ }^{5}$ Departament d'Ecologia. Universitat de Barcelona. Diagonal, 645. 08028 Barcelona.

${ }^{6}$ Departamento de Biología Vegetal y Ecología. Universidad de Almería. Cañada de San Urbano, s/n. 04120

Almería.

${ }^{7}$ Departamento de Biología Vegetal. Universidad de Granada. Campus Universitario de Fuentenueva. 18701 Granada.

\section{RESUMEN}

Se ha aplicado el QBR (índice de calidad del bosque de ribera) a 157 estaciones de muestreo localizadas en ríos pertenecientes a 12 cuencas diferentes del ámbito mediterráneo español. Los resultados indican que más del $34 \%$ de las estaciones muestran valores de calidad buenos y muy buenos (QBR $>75)$, mientras que el $45 \%$ presenta valores de mala y pésima calidad $($ QBR $<50)$. Según una tipología previa establecida para clasificar las estaciones de muestreo, los valores de mayor calidad de QBR se dan en estaciones de cabecera de cuencas calcáreas y en las zonas de karst. Los tipos denominados temporales y ramblas presentan los valores más bajos de calidad, o no tiene representantes de máxima calidad.

La ausencia o escasez de bosques de riberas de máxima calidad en las cuencas del sur peninsular puede explicarse por el gradiente de aridez que se establece desde el norte hacia el sur. Además existe un evidente deterioro de las riberas desde las cabeceras a las desembocaduras de los ríos, de manera que ambos factores influyen en el estado actual de las riberas de los ríos mediterráneos ibéricos. Finalmente se discute la utilidad y limitaciones del QBR en ríos sometidos a situaciones de estrés hídrico o ambiental.

Palabras clave: calidad de las riberas, índice QBR, ríos mediterráneos, Directiva Marco del Agua

\begin{abstract}
An index of riparian quality (QBR) was applied to 157 sampling sites in streams of twelve Mediterranean basins. Our results show that approximately $34 \%$ of the total number of sampling sites had riparian habitat in natural conditions and of good quality $(Q B R>75)$, while $45 \%$ had experienced strong alteration of natural conditions, and quality of the riparian was poor $(Q B R<50)$. The good quality $Q B R$ sites were found in sampling areas located in upstream areas of calcareous basins and in karst zones. The stream type called "ramblas" and the "temporary streams" included many sampling sites with riparian of very bad quality. For these types of stream, no sampling site with riparian habitat in "natural condition" was found.

Riparian high quality sites were scarcer in sites located to the south of the Iberian Peninsula. This may be explained by the increasing aridity gradient towards the South. In the catchments located in the south of the Iberian Peninsula, the riparian vegetation typical of the areas was usually absent. Moreover, we have identified a strong degradation of the riparian from the head to the mouth of all streams studied. Therefore, both aridity and human induced degradation explain the present quality status of riparian vegetation in the catchments studied. Finally, we discuss the scope of use and limitations of the QBR index in streams experiencing extreme hydrological and environmental stress.
\end{abstract}

Key words: Riparian vegetation quality, QBR index, mediterranean streams, Water Framework Directive 


\section{INTRODUCCIÓN}

Entre los aspectos a considerar para cuantificar y calificar la "calidad ecológica" de los ecosistemas acuáticos, propuestos por la Directiva Marco del Agua (DMA) (D.O.C.E., 2000), las riberas constituyen uno de los más importantes. Las razones son obvias y obedecen por una parte a sus valores naturales (elevada riqueza y diversidad florística y faunística) (Girel \& Manneville, 1998; Ward, 1998); a la posibilidad de diversificación del paisaje incorporando elementos biogeográficos en cierta manera anómalos (González Bernáldez, 1988); a su capacidad para incidir sobre la calidad ambiental del ecosistema acuático que rodea, a través del control de la temperatura del agua (Beschta et al., 1997), de la entrada de materiales orgánicos externos (e.g., Fisher \& Likens, 1973) y de los nutrientes (Schade et al., 2001 ; 2002) e incluso por su capacidad para diseñar microambientes terrestres (Brosofske et al., 1997) y acuáticos utilizados para diversas funciones por los organismos (Gregory et al., 1991). Además, la vegetación ribereña juega un papel esencial en la retención y atenuación de los efectos destructores de las avenidas de agua (Decamps, 1996).

Como zona de transición o interfase (Mitsch et al., 1994) ha sido muy estudiado su función de "filtro" y, en cierta medida de sistema "depurador" (Osborne \& Kovacic, 1993; Haycock et al., 1996), aunque queda mucho por conocer sobre su función como "comunicador" entre el ecosistema acuático y su entorno terrestre y como "corredor" en el sentido longitudinal (Burbrink et al., 1998; Naiman et al., 2000; Puth \& Wilson, 2001). Recientemente se le está prestando mucha atención a las relaciones complejas que se establecen entre el flujo natural de agua y su variabilidad anual sobre la estructura y organización de la vegetación de los bosques ribereños (Pettit et al., 2001; Shafroth et al., 2002).

Todos estos valores y funciones que ostentan los bosques de ribera les hacen excelentes indicadores de la gestión del territorio y este es el sentido de su inclusión como elemento clave para la calificación del estado ecológico de los ríos. Sin embargo, las riberas siempre han estado en conflicto con el hombre, puesto que en ellas se ha desarrollado todo tipo de actividades con distinto grado de impacto (agricultura de regadío, ganadería-pastoreo, vías de comunicación, etc) (Chaney et al., 1990). Hoy día las riberas se encuentran en un estado importante de degradación general, lo cual ha generado una fructífera línea de trabajo cuyo objetivo es diseñar y ensayar técnicas de restauración (e.g., Campbell et al., 1998; González del Tánago \& García de Jalón, 1998; Molles et al., 1998; González del Tánago, 1999).

No existen muchas propuestas para cuantificar la calidad ambiental de las riberas utilizando índices de fácil manejo y de aplicación sencilla, así que Munné et al. (1998; 2003), propusieron el QBR (Índice de Calidad del Bosque de Ribera) que en cuatro bloques recoge distintos componentes y atributos de las riberas: - cubierta vegetal, -estructura de la vegetación, - naturalidad y complejidad del bosque ribereño y - grado de alteración del canal fluvial. Los valores del índice se distribuyen en cinco rangos de calidad ( $>95$ : estado natural; 90-75: calidad buena; 7055: calidad aceptable; 30-50: calidad mala; $<25$ : calidad pésima) El índice ya ha sido aplicado en distintos ríos españoles (González del Tánago \& Antón, 1998; 2000; Carrascosa \& Munné, 2000; Casas et al., 2000; Prat et al., 1999; Suárez \& Vidal-Abarca, 2000; Zamora-Muñoz et al., 2000; Martínez \& Lozano, en prensa) y portugueses (Gonçalves, 2001) con buenos resultados.

En términos generales, los ríos mediterráneos se caracterizan por su variabilidad hídrica espacio-temporal lo cual les lleva a tolerar desde periodos de sequía importantes y relativamente largos hasta avenidas de agua puntuales de distinta intensidad (Gasith \& Resh, 1999). Las riberas están sometidas a estas perturbaciones y su estado natural refleja en buena medida el régimen hídrico que prevalece. La búsqueda de la calidad potencial máxima de las riberas dentro de la variabilidad que representan los ríos mediterráneos es un objetivo a conseguir a largo plazo.

Desde el año 1999, seis grupos de investigación de las universidades de Barcelona, Granada, 
Almería, Illes Balears-Vigo, Murcia y el CEDEX de Madrid, reunidos bajo el nombre de GUADALMED, vienen realizando un estudio extensivo conjunto para establecer los rangos de calidad de los ríos mediterráneos ibéricos que, finalmente lleve a la calificación del estado ecológico actual de estos sistemas. Entre los distintos componentes físicos y biológicos que se analizan (hábitat, macrófitos y macroalgas, macroinvertebrados, etc) las riberas se han estudiado aplicando el QBR. Así el principal objetivo de este trabajo es analizar la calidad de los bosques de ribera de los ríos mediterráneos ibéricos utilizando el QBR y su variación en función de distintos parámetros fisiográficos y ambientales. Finalmente se discute la validez del índice en regiones de fuerte contraste ambiental como ocurre en la vertiente mediterránea.

\section{EL ÁREA DE ESTUdIO}

El estudio se llevó a cabo en distintos ríos pertenecientes a las cuencas del Besòs, Llobregat, Mijares, Turia, Júcar, Sóller, Pollença, Segura, Aguas, Almanzora, Adra y Guadalfeo, recogiendo la totalidad del gradiente ambiental de aridez característico del mediterráneo que se puede establecer desde el noreste peninsular más fresco y húmedo (precipitación anual $>600 \mathrm{~mm}$ ) hasta el sureste más cálido y seco (precipitación anual $<300 \mathrm{~mm}$ ) (VidalAbarca et al., 1992).

El régimen hidrológico mediterráneo general, está muy alterado en la mayoría de los ríos estudiados por la extracción exhaustiva del agua para diversas actividades, desde la industria y uso urbano, hasta el agrícola de forma mayoritaria. Hay un predominio de materiales sedimentarios en el territorio, lo cual influye en la calidad natural del agua (aportes de sales de distinta tipología). Existe, además un problema general de pérdida de calidad del agua como consecuencia de los vertidos orgánicos e industriales a la mayoría de los ríos estudiados (Prat, 1996). Para un análisis más detallado de las cuencas incluidas en este estudio ver Robles et al. (este volumen).

\section{METODOLOGÍA}

Se seleccionaron un total de 157 estaciones de muestreo en ríos de las 12 cuencas estudiadas teniendo en cuenta la regionalización del territorio en base a distintos parámetros fisiográficos y ambientales (Bonada et al., este volumen).

En general, las estaciones se seleccionaron teniendo en cuenta su situación en los diferentes tramos del río y en las diferentes cuencas. De igual manera, se seleccionaron estaciones en aquellos sistemas fluviales más peculiares y extremos del mediterráneo como ramblas y ríos salinos. Para una descripción detallada de las cuencas y los puntos de muestreo, ver Robles et al. (este volumen).

El periodo de muestreo abarca desde la primavera de 1999 hasta el invierno de 2000. El protocolo seguido para la aplicación del índice QBR se describe en Munné et al. $(1998 ; 2003)$ y de forma más sencilla en Jáimez-Cuéllar et al. (este volumen). Dado que el índice QBR no está sujeto a una variabilidad estacional puesto que utiliza como indicador biológico la vegetación arbórea, arbustiva y el matorral perenne, únicamente se ha utilizado un valor del índice para el tratamiento de los datos. En caso de que tener varios valores del índice se seleccionó el más frecuente y en caso de que hubiera dos valores se seleccionó el mayor. De cualquier manera nunca hubo cambio en el rango de calidad del QBR cuando existían varias estimaciones del índice.

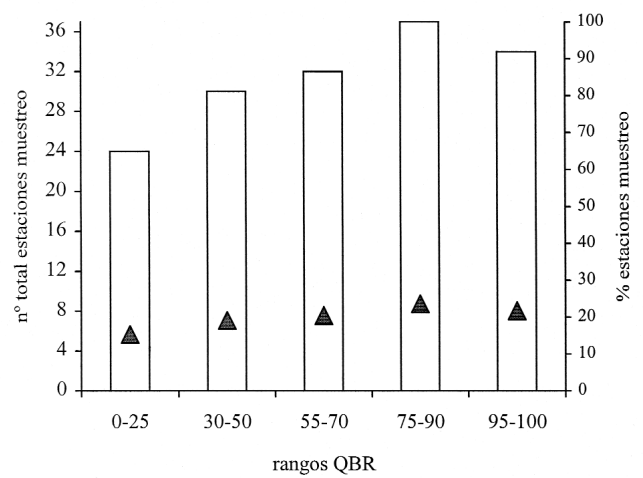

Figura 1. Distribución del número total de estaciones de muestreo y \% que representan según su rango de calidad para el índice de riberas QBR. Number and percentage of sampling sites over the total number sampled in each quality class according to the $Q B R$ index. 

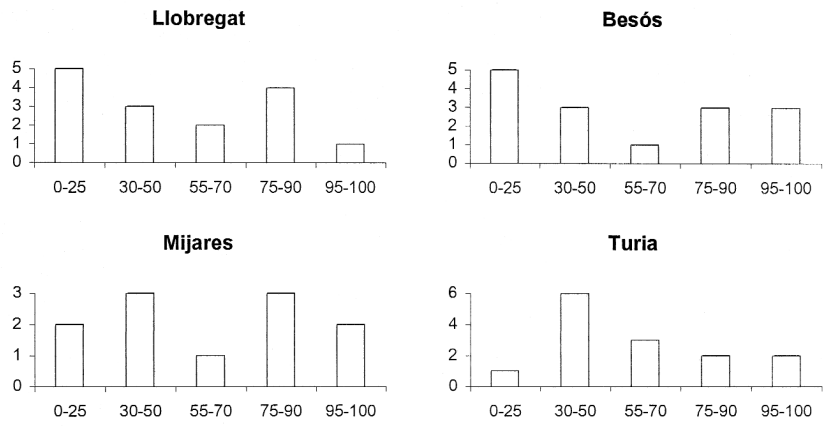

Júcar
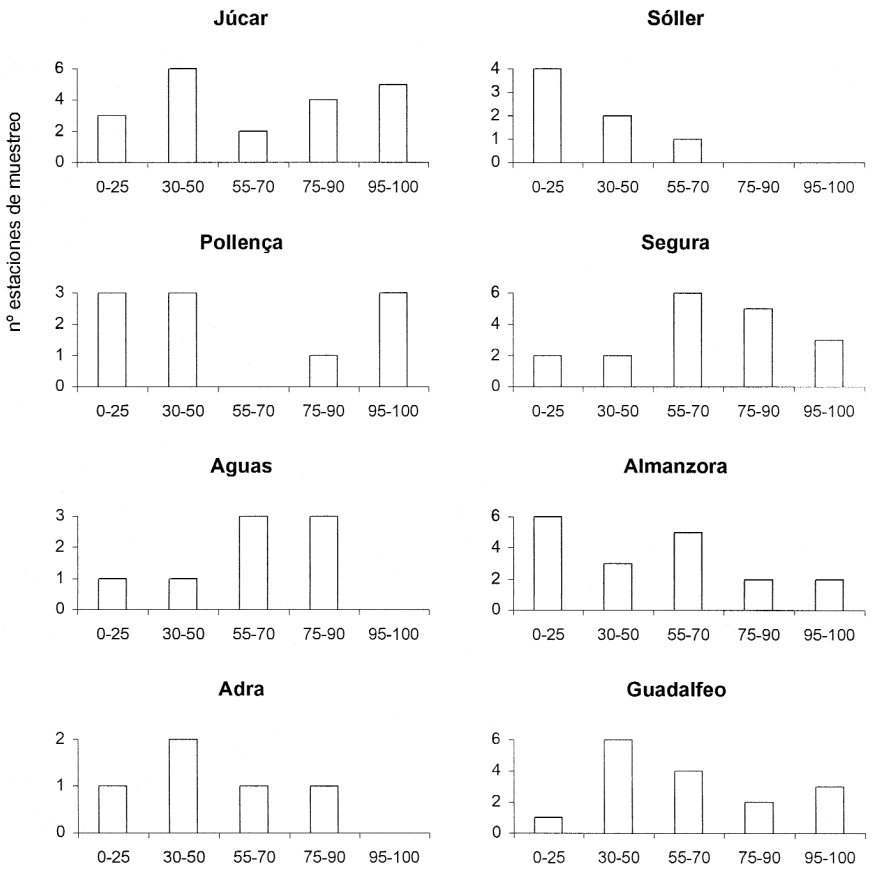

rango QBR

Figura 2. Distribución de las estaciones de muestreo de las distintas cuencas estudiadas según su rango de calidad para el índice de riberas QBR. Distribution of sampling sites grouped by basin in quality classes according to the QBR index.

Para explorar de forma conjunta los datos obtenidos se aplicó un análisis de componentes principales (PCA) del paquete estadístico XLSTAT (Fahmy, 1998) sobre una matriz de 157 estaciones de muestreo por 8 variables: el índice QBR, altitud, y el grado de cobertura, estructura de la cubierta vegetal, naturalidad de la ribera, grado de alteración, tipo geomorfológico y puntuación del tipo, todas ellas derivadas del propio índice. De esta manera se pretende analizar si alguna de ellas tiene mayor influencia o explica mejor las variaciones en la calidad de las riberas.

\section{RESULTADOS}

En la figura 1 se muestra la distribución del número total de estaciones de muestreo y porcentaje que representan, según su rango de calidad para el índice de riberas. También se muestra el mismo tipo de relación para cada una de las cuencas estudiadas (Fig. 2).

Más del $34 \%$ de las estaciones muestreadas presentan valores buenos de calidad (QBR $>75)$, aunque las de mala y pésima calidad $(\mathrm{QBR}<50)$ constituyen el $45.3 \%$ (71 estaciones de muestreo). 


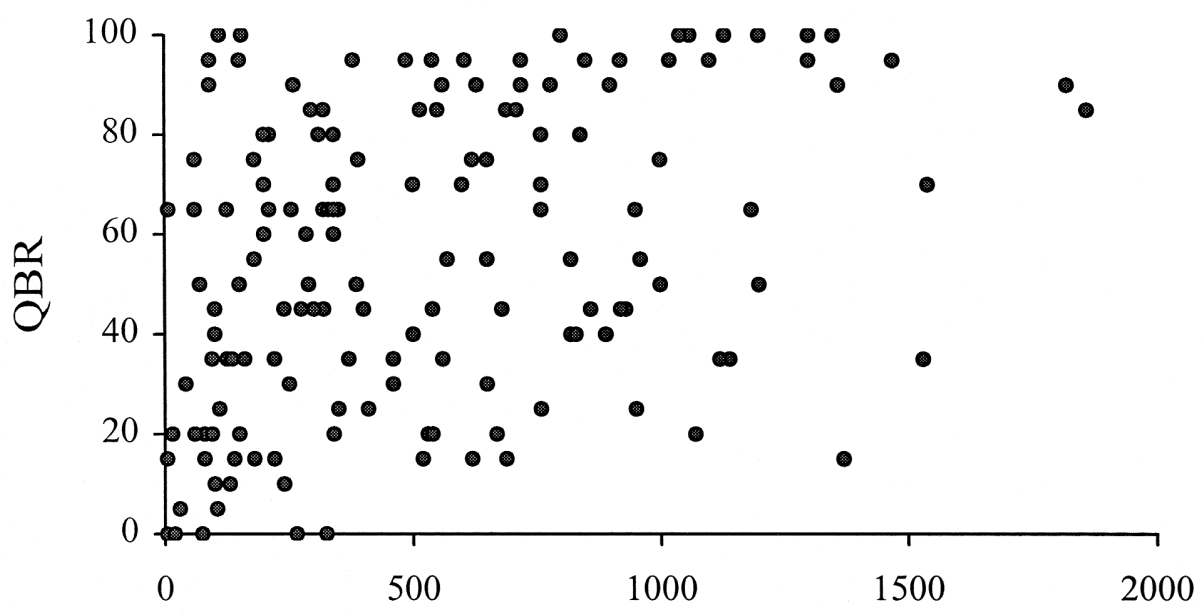

Altitud (msnm)

Figura 3. Representación gráfica de los valores de QBR frente a la altitud (m) de las estaciones de muestreo. QBR values and altitude (m) of the studied sampling sites.

La distribución espacial de los valores del índice apuntan hacia una disminución de la calidad desde la cabecera a la desembocadura (coeficiente de correlación entre altitud y valor de QBR, $\mathrm{r}=0.388, \mathrm{p}<0.001)$ lo cual no es extraño dado que, en términos generales, la degradación de las riberas es más intensa en la partes bajas de los ríos que en las cabeceras debido, entre otros a la mayor presión humana en estas zonas (Ibero et al., 1996).

Sin embargo, la relación entre la altitud y los valores del índice de calidad de ribera (Fig. 3) revela la distribución relativamente uniforme de las estaciones de muestreo en todos los rangos de calidad del QBR, es decir la degradación afecta tanto a los tramos de cabecera como a los medios y bajos de los ríos estudiados.

El análisis de los rangos del QBR por cuencas revela situaciones dignas de comentar (Fig. 2). En términos generales, el gradiente de aridez de la región mediterránea parece quedar reflejado en la casi ausencia de estaciones en "estado natural" (QBR > 95) en las cuencas más sureñas como la de los ríos Adra y Aguas, en las que probablemente se dan las condiciones climáticas más extremas. Esta regla no se cumple para ríos que dependen de aportes externos de agua como el Guadalfeo cuyo régimen hídrico es torrencial, pero dependiente de un aporte nival desde Sierra Nevada (Alba-Tercedor \& Jiménez, 1985) o el río Segura que nace fuera del ámbito árido mediterráneo (Vidal-Abarca et al., 1987; González del Tánago et al., 1995).

Otro caso es el de los ríos de Mallorca, principalmente agrícolas, donde dominan los cultivos en terraza debido a las fuertes pendientes de las laderas en los tramos medios y bajos. En las cabeceras, muy cerradas, el agua circula escasamente uno o dos meses al año, lo cual impide el desarrollo de un bosque completamente estructurado (Álvarez et al., 2001; 2002).

En general, para casi todas las cuencas, alrededor del $50 \%$ de las estaciones de muestreo presentan mala y pésima calidad de riberas (Fig. 2). Las excepciones que se observan, como por ejemplo en la cuenca del Segura, en la que no se alcanza el $25 \%$ de las estaciones muestreadas, se debe a la exclusión, a priori, de puntos de muestreo donde la degradación del sistema fluvial era muy evidente. En otras ocasiones, como ocurre en el río Aguas, el régimen hidrológico extremo impidió establecer estaciones de muestreo en los tramos más bajos que permanentemente están secos y tienen las riberas más degradadas.

Cuando se analizan los rangos del QBR por tipología de estaciones de muestreo, tal como se definen en Bonada et al. (este volumen) 
(Figura 4), se observan como los tipos que presentan la mayor cantidad de estaciones de muestreo en el rango de pésima calidad $(\mathrm{QBR}<25)$ son los denominados temporales y los ríos de cuencas silíceas en tramos mediosbajos (Sil/MB). Por el contrario, los ríos situados en zonas kársticas (Karst) y los localizados en las cabeceras de las cuencas calcáreas $(\mathrm{Cal} / \mathrm{Cab})$, presentan el mayor porcentaje de estaciones en el rango de máxima calidad (QBR > 95). En el tipo ramblas, en los ríos de cuencas silíceas en los tramos medios-bajos (Sil/MB) y en las cuencas calcáreas grandes $(\mathrm{Cal} / \mathrm{GB})$, no se tienen estaciones con valores para el índice que indiquen máxima calidad.

El análisis de componentes principales (PCA) realizado sobre la matriz de 157 estaciones de muestreo, explica el $83 \%$ del total de la varianza en los tres primeros ejes (Tabla 1). El primero está definido positivamente por el QBR y los cuatro apartados del mismo: cobertura, estructura, naturalidad y grado de alteración, ordenando así las estaciones según la calidad de sus riberas. El segundo eje está definido positivamente por la puntuación del tipo y negativamente por el tipo geomorfológico, discriminando las estaciones de cabecera de las de los tramos más bajos. Por último el tercer eje, quedó definido por la altitud expresando, en cierta medida su independencia con respecto a la calidad de las riberas. Como ya se ha comentado existe una correlación significativa y positiva entre altitud y QBR aunque el valor de coeficiente $r$ es muy bajo $(\mathrm{r}=0.388)$ (Fig. 3).

La ordenación de las muestras en el plano definido por los ejes I y II se presenta en la figura 5. En una primera interpretación, el análisis organiza las estaciones en tres bandas separando las localizadas en las cabeceras y tramos altos, de las situadas en los tramos medios y medios-bajos y finalmente en los tramos más bajos y desembocaduras. En cada uno de estos grupos las estaciones cubren el total de rangos de calidad del QBR. De hecho cuando las estaciones se interpretan en términos de rangos de calidad para este índice (Fig. 6), las bandas siguen la dirección perpendicular a este supuesto gradiente altitudinal. La única limitación, como ya se ha comentado, es la ausencia de estaciones de buena o máxima calidad (verdes y azules, respectivamente) en los tramos bajos de muchos de los ríos estudiados (Fig. 4).

Tabla 1. Resultados del Análisis de Componentes Principales efectuado sobre la matriz de datos: porcentaje de varianza explicada por cada uno de los tres primeros componentes y correlaciones obtenidas entre las variables iniciales y cada uno de ellos (los valores menores de 0.25 no se incluyen). Cumulative variance and eigenvalues for the first three axes of the PCA performed on the whole data matrix. \% variance explained by the three first components and the correlation coefficients between initial variables and between axes are also shown (the eigenvalues less than 0.25 have not been included).

\begin{tabular}{lccc}
\hline & $\mathbf{1}$ & $\mathbf{2}$ & $\mathbf{3}$ \\
\hline Varianza explicada (\%) & 53.57 & 18.97 & 10.42 \\
Varianza acumulada (\%) & 53.57 & 72.54 & 82.96 \\
& & & \\
& factor $\mathbf{1}$ & factor $\mathbf{2}$ & $\mathbf{f a c t o r} \mathbf{3}$ \\
\hline Altitud & 0.5272 & & 0.8071 \\
QBR & 0.9550 & -0.2793 & -0.3681 \\
Cobertura vegetal & 0.7951 & -0.3374 & \\
Estructura vegetación & 0.7920 & -0.4434 & \\
Calidad ribera & 0.7961 & 0.7652 & \\
Grado naturalidad & 0.6906 & -0.7028 & \\
Puntuación del tipo & 0.5476 & & \\
Tipo geomorfológico & -0.6527 & & \\
\hline
\end{tabular}


Con el fin de simplificar la interpretación del análisis según la tipología de estaciones de muestreo, en la figura 7 se ha representado el valor medio de las coordenadas de todas las estaciones pertenecientes a cada uno de los tipos definidos. Aquellos que mayor cantidad de estaciones de máxima calidad presentan para el QBR, son los que incluyen las cabeceras tanto de cuencas silíceas como calcáreas, y

Cal/Cab

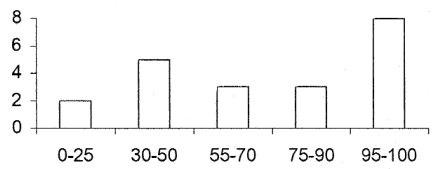

Cal/MB

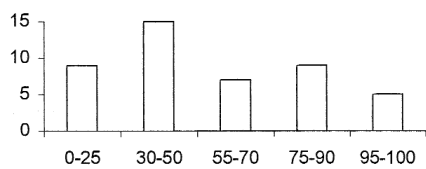

Llanura Aluvial

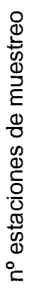

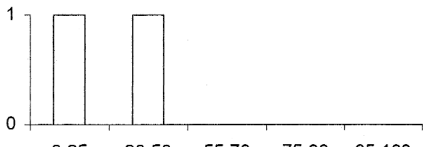

sil/cab

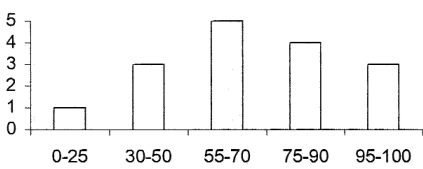

Temporales

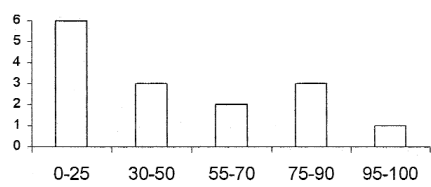

rango $Q B R$ los situados en zonas de karst. Por el contrario, el tipo llanuras aluviales y cuencas silíceas medias y bajas (Sil/MB), e incluso las ramblas, son los que incluyen mayor numero de estaciones de muy baja calidad. Los ríos temporales quedan en una situación intermedia de calidad, aunque en general, parece que se localizan en las zonas de cabeceras de las cuencas estudiadas.

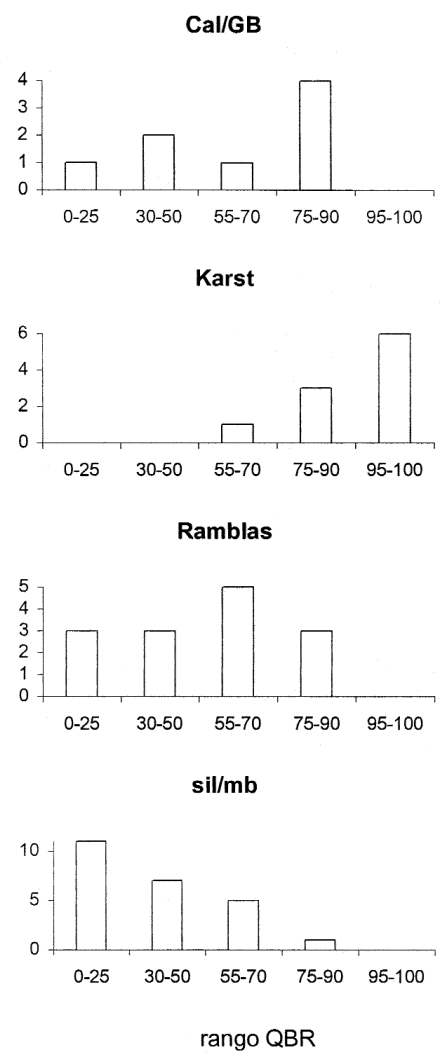

Figura 4. Distribución de las estaciones de muestreo por tipos según su rango de calidad para el índice de riberas QBR. (Cal/Cab: cabeceras de cuencas calcáreas; Cal/GB: tramos bajos de cuencas calcáreas grandes; Cal/MB: tramos medios y bajos de cuencas calcáreas; Karst: karst; Llanura Aluvial: llanura aluvial; Ramblas: ramblas; Sil/Cab: cabeceras de cuencas silíceas; Sil/MB: tramos medios y bajos de cuencas silíceas ; Temporales: temporales). Distribution of sampling sites grouped into riparian quality classes according to ecotype. (Cal/Cab = headwater of calcareous basins; Cal $/ G B=$ Large calcareous basins; Cal/MB $=$ Medium and low reaches of calcareous basins; Llanura aluvial = floodplains; Karst = karstic basins; Ramblas = "ramblas"; Sil/Cab = headwater of siliceous basins; Sil/MB = Medium and low reaches of siliceous basins; Temporales = Temporary streams). 


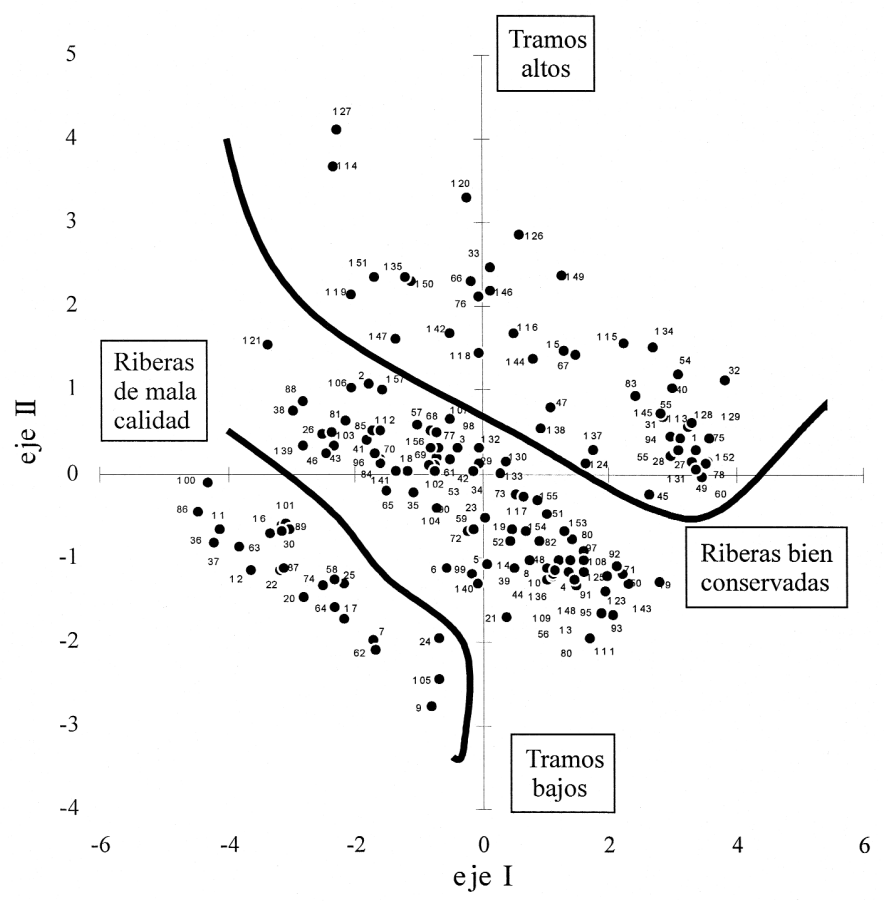

Figura 5. Ordenación de las estaciones de muestreo en el plano definido por los ejes I y II de análisis de componentes principales (PCA). Plot of sampling sites in the space defined by the first two PCA components.

\section{DISCUSION}

Un índice de calidad debe ser suficientemente amplio como para recoger la variabilidad de aquel parámetro o factor del que se quiere valorar su calidad (Karr, 1999). El QBR ha sido probado en diferentes cuencas y ajustado para dos situaciones concretas. Así, Carrascosa \& Munné (2000) establecen la necesidad de no penalizar la ausencia de un estrato arbóreo en ríos de alta montaña de valles cerrados, en el apartado relativo a la estructura de la vegetación y Suárez \& Vidal-Abarca (2000), señalan la conveniencia de considerar el estrato arbustivo bien desarrollado como estrato arbóreo en ríos del sureste peninsular sometidos a situaciones de estrés ambiental (por ejemplo, avenidas), y la necesidad de incorporar la diversidad y el estado de conservación de la vegetación más xerófila en ramblas y tramos de cauces que permanecen secos durante gran parte de ciclo hidrológico.
En este trabajo, la aplicación del QBR para valorar la calidad de las riberas de los ríos mediterráneos tiene como ventaja sobre otros estudios, la cantidad de estaciones de muestreo (157), la variedad (9 tipos de ríos diferentes) y las veces (al menos todos los ríos tienen dos valores de QBR) en que se calculó el índice, lo cual permite valorar, con un cierto grado de objetividad los resultados y posibilidades del mismo.

La ausencia de estaciones en "estado natural" (máxima calidad; QBR > 95) conforme aumenta el grado de aridez de norte a sur de la cuenca mediterránea, podría constituir una de las limitaciones del índice de calidad QBR. La vegetación ribereña está ausente o en estado muy precario en sistemas de evacuación rápida como las ramblas típicas del ámbito mediterráneo, desconectadas de sistemas acuíferos de cierta entidad, por lo que permanecen secas durante la mayor parte del año y solo transportan agua de evacuación muy rápida tras las fuertes tormentas 


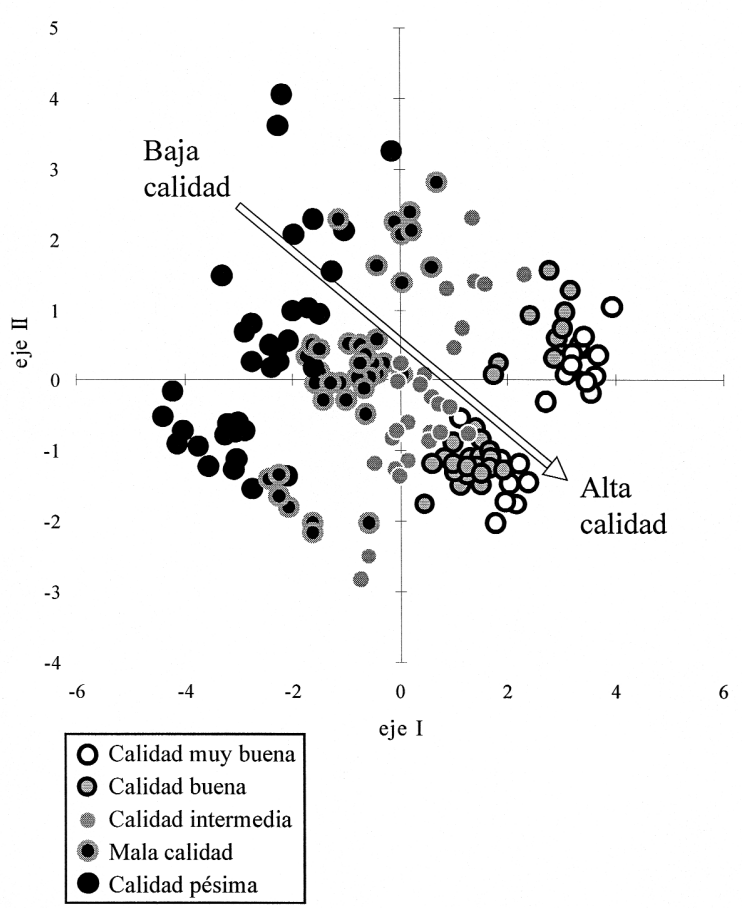

Figura 6. Interpretación del PCA en función de los rangos de calidad del índice QBR. Interpretation of PCA results according to riparian quality classes.

(Vidal-Abarca et al., 1996). Evidentemente para este tipo de sistema no es posible realizar el cálculo de la calidad de riberas y aplicar el índice QBR directamente tal y como se establecido por Munné et al. (1998).

Sin embargo, el grado de aridez lleva implícito anomalías hídricas que sí afectan a la vegetación ribereña de forma decisiva. Así por ejemplo, las avenidas de agua son tanto más impredecibles conforme nos dirigimos al sur (Vidal-Abarca, 1990; Comín \& Williams, 1994) lo cual puede afectar tanto a la estructura, como al porcentaje de cobertura vegetal (Pettit et al., 2001). En efecto, las avenidas remueven sedimentos y eliminan la vegetación ribereña (Van der Sman et al., 1993; Swanson et al., 1998; Boulton \& Brock, 1999) imposibilitando la sucesión hacia un bosque ribereño típico (Decamps, 1996). Pero además otros sistemas fluviales como los ríos y ramblas que transpor- tan aguas salinas constituyen anomalías, en este caso fisico-químicas, que altera y evidentemente condiciona la estructura y el grado de cobertura de la vegetación riparia (Moreno et al., 1996; Suárez \& Vidal-Abarca, 2000).

Los valores máximos del apartado de grado de cobertura vegetal en el QBR solo se pueden conseguir si la vegetación (excluidas las plantas anuales) cubre más del $80 \%$ de la ribera, al igual que para obtener los valores más altos en el apartado de la estructura, es necesario que existan árboles o arbustos (Munné et al., 1998). Únicamente con los helófitos no es posible alcanzan la máxima puntuación. Ambos apartados están condicionados por las situaciones antes comentadas lo cual impide, en muchas estaciones de muestreo, alcanzar el valor necesario del QBR para ser calificados como "estado natural". Esta situación es interesante sobre todo cuando se pretende adoptar técnicas de restaura- 
ción de riberas en tramos o cauces donde, de forma más o menos natural, la vegetación está condicionada por factores estresantes (Boulton \& Brock, 1999). De esta manera, al igual que existe un patrón que identifica perfectamente la estructura y el porcentaje de cobertura que debe cumplir un "bosque ribereño típico", resulta imprescindible establecer otro $u$ otros patrones o estándares de "bosquete ribereño" (tipo y variedad de plantas) y el porcentaje de cobertura vegetal máximo posible, en situaciones reconocidas de estrés hídrico o ambiental.

Ante esto no resulta extraño que la única correlación no significativa se establezca entre la altitud y el apartado del QBR relativo a la cobertura vegetal (para el resto de apartados y para el QBR, las correlaciones son significativas). El porcentaje o nivel de ocupación de la vegetación ribereña, no siempre responde a gradientes ambientales más o menos ligados a la altitud, por ejemplo muchas actividades humanas son incompatibles con un buen nivel de ocupación vegetal.

En general, no existen correlaciones significativas entre los valores del QBR y sus apartados y otras variables ambientales analizadas (área de la cuenca, porcentaje de tipos de sustratos geológicos diferentes, etc.). Únicamente existe una relación significativa y negativa $(\mathrm{r}=$ $-0.24, \mathrm{p}<0.01$ ) entre el área de la cuenca y el grado de naturalidad, lo cual parece indicar que la mayor degradación del canal fluvial se produce en las cuencas más grandes. Pero más interesante resulta las correlaciones que se establecen entre la calidad de la cubierta vegetal y el porcentaje de sustrato calcáreo que presenta la cuenca $(\mathrm{r}=0.247 ; \mathrm{p}<0.01)$ y el porcentaje de sustrato margoso $(r=-0.247 ; p<0.01)$. Al ser en sentido opuesto (positivo en el primer caso y negativo en el segundo) parece indicar, o bien que las mayores agresiones (basuras, especies arbóreas introducidas o estructuras construidas por el hombre) se producen en las cuencas donde dominan las margas, fundamentalmente en las cuencas del Segura, Adra y Almanzora, o bien que el gradiente de aridez o de estrés hídrico que tiene su mayor exponente en el sureste ibérico, se manifiesta en una calidad de la cubierta vegetal menor (por ejemplo, por la ausencia de árboles autóctonos).

Los resultados del PCA reflejan que las situaciones de mejor calidad en las riberas se da en las estaciones de muestreo localizadas en zonas de cabecera, difícilmente accesibles que sustentan un bosque ribereño bien estructurado. En general, las estaciones de muestreo de los distintos ríos no siguen una ordenación geográfica definida por cuencas. No ocurre lo mismo cuando se interpretan en términos de rangos de calidad (ver Fig. 6) o en relación a la tipología establecida (Fig. 7). En ambos casos se detectan patrones de fácil identificación como en el primer caso, aunque para el segundo son más discutibles. Dado que para la definición de los tipos no fue posible utilizar algún parámetro hidrológico indicador del grado de temporalidad de agua (Bonada et al., este volumen), es posible que no hayan quedado bien delimitados los requisitos que permitan discriminar los tipos denominados: ramblas, temporales e incluso el tipo karst. Por ejemplo, la estación SE04 (Río Mundo en los Chorros; cuenca del Segura) está mantenida por un karst pero se seca en verano, al menos dos meses, lo cual hace que pueda incorporarse a cualquiera de estos dos tipos. Además, muchas estaciones tipificadas como ramblas son también temporales (por ejemplo la estación SE15, rambla del Puerto del Garruchal; cuenca del Segura, se seca en verano) lo cuál hace que muchas de ellas puedan participar de ambos tipos.

En definitiva, el índice QBR es útil para establecer el nivel de calidad de las riberas de los ríos mediterráneos. Únicamente hay que considerar ciertas precauciones cuando se estudian ríos o sistemas fluviales sometidos a cualquier estrés ambiental natural u originado por el hombre que imposibilite la formación de un bosque de ribera y que, de esta manera, podría infravalorar su estado de conservación. Para ello, sería deseable establecer el bosque ribereño potencial y posible en función de cada tipo de río y de estrés natural. En la segunda fase del proyecto GUADALMED se incidirá en este aspecto. 


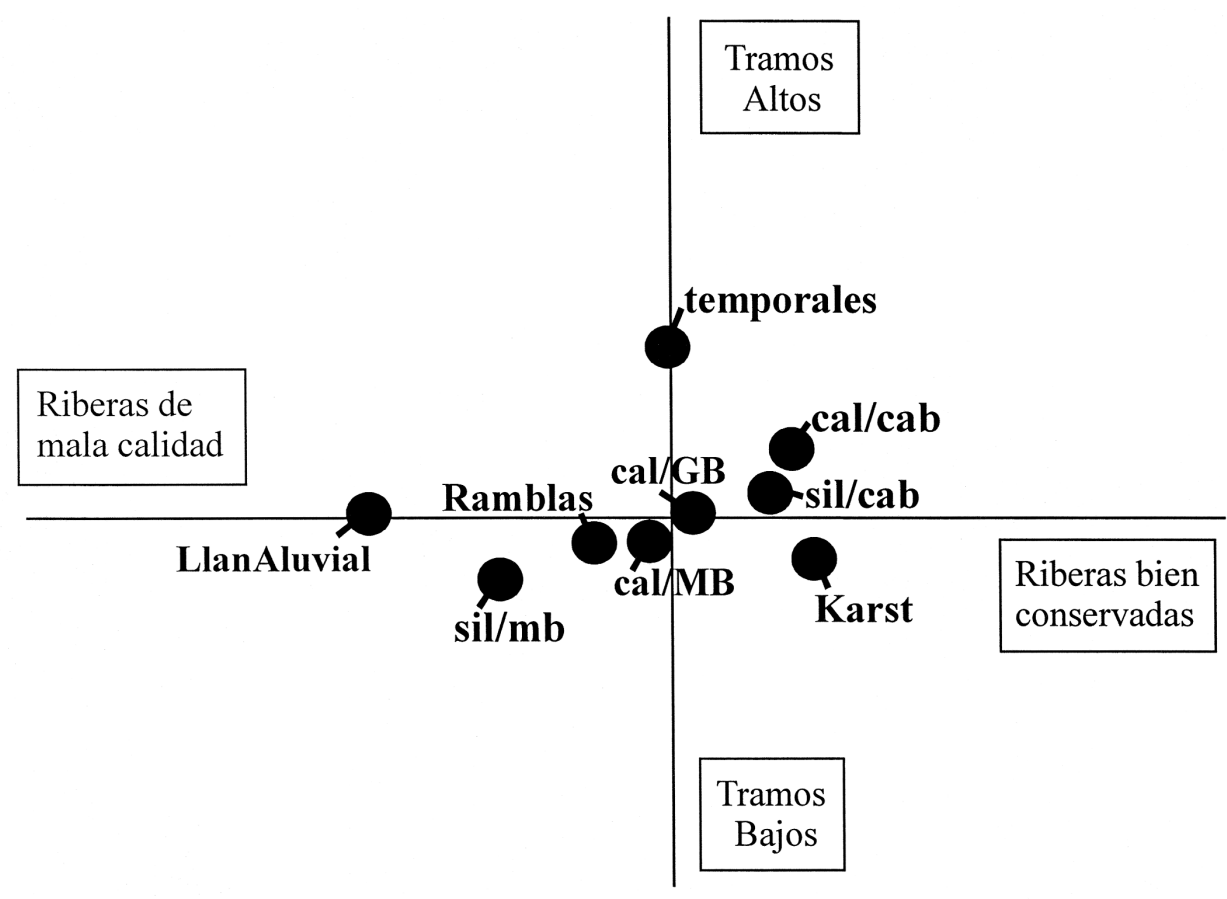

Figura 7. Valores medios de las coordenadas del PCA para las estaciones de muestreo agrupadas por tipos (para la clave de los tipos ver pie de la figura 4). Mean values for PCA scores of sampling sites grouped by ecotype (for a key, see figure 4).

\section{AGRADECIMIENTOS}

Este trabajo se ha realizado mediante la financiación de los proyectos HID98-0323-C05 y REN2001-3438-C07 del Ministerio de Ciencia y Tecnología y PLP/10/FS/97 de la Fundación Séneca de la CARM. Nuestro especial agradecimiento al Area de Coordinación y Aplicaciones Tecnológicas de la D.G.O.H. del Ministerio de Medio Ambiente, al Àrea de Medi Ambient de la Diputació de Barcelona, a la Delegación de Granada de la Consejería de Medio Ambiente de la Junta de Andalucía y a la Agencia Catalana del Aigua por su apoyo.

\section{BIBLIOGRAFÍA}

ALBA-TERCEDOR, J. \& F. JIMÉNEZ. 1985. Evaluación de las variaciones estacionales de la calidad de las aguas del río Guadalfeo basada en el estudio de las comunidades de macroinvertebrados acuáticos y de los factores fisico-químicos.
Proyecto LUCDEME III. Monografías 48. ICONA. $91 \mathrm{pp}$.

ÁlVAREZ, M., I. PARDO, G. MOYA, G. RAMON \& A. MARTINEZ-TABERNER. 2001. Invertebrate communities in temporary streams of the island of Majorca: a comparison of catchments with different land use. Limnetica, 20: 255-266

ÁlVAREZ, M., I. PARDO, G. MOYÁ, A. MARTINEZ-TABERNER \& G. RAMON. 2002. Abiotic and biotic factors determining invertebrate community structure in the torrents of Majorca. Verh. Internat. Verein. Limnol., 28: 1223-1228.

BESCHTA, R. L., R. E. BILBY, G. W. BROWN, L. B. HOLTBY \& T. D. HOFSTRA. 1987. Stream temperature and aquatic habitat: fisheries and forestry interactions. In: Streamside Management: Forestry and Fishery Interactions. E.O. Salo, T.W. Cundy (eds.): 191-232. Institute of Forest Resources, University of Washington, Contribution $\mathrm{n}^{\circ} 57$, Washington, USA.

BONADA, N., N. PRAT, A. MUNNÉ, M. RIERADEVALL，J. ALBA-TERCEDOR， M. ÁLVAREZ, J. AVILÉS, J. CASAS, P. JÁIMEZCUÉLLAR, A. MELLADO, G. MOYÀ, I. PARDO, 
S. ROBLES, G. RAMON, M.L. SUÁREZ, M. TORO, M.R. VIDAL-ABARCA, S. VIVAS \& C. ZAMORA-MUÑOZ. 2002. Ensayo de una tipología de las cuencas mediterráneas del proyecto GUADALMED siguiendo las directrices de la Directiva Marco del Agua. Limnetica, 21: 77-98.

BOULTON, A. \& M. A. BROCK. 1999. Australian Freshwater Ecology. Processes and management. Australia: Gleneagles Pub.

BROSOFSKE, K. D., J. CHEN, R. J. NAIMAN \& J. F. FRANKLIN. 1997. Harvesting effects on microclimatic gradients from small streams to uplands in western Washington. Ecol. Appl., 7: 118-1200.

BURBRINK F. T., C. A. PHILLIPS \& E. J. HESKE. 1998. A riparian zone in southern Illinois as a potential dispersal corridor for reptiles and amphibians. Biol. Conserv., 86: 107-115.

CAMPBELL I. C., P .J. BOON, B. L. MADSEN \& K. W. CUMMINS. 1998. Objectives and approaches in lotic and riparian restoration projects. Verh. Internat. Verein. Limnol., 26: 1295-1302.

CARRASCOSA, V. \& A. MUNNÉ, 2000. Qualificació dels boscos de ribera andorrans. Adaptació de l'index QBR als d'alta muntanya: Habitats-Centre de Biodiversitat (IEA), 1: 4-13.

CASAS, J. J., M. SALINAS, S. VIVAS, M. BAYO \& D. LÓPEZ. 2000. Vegetación de las riberas de dos cuencas semiáridas (ríos Almanzora y Aguas, Almería): Relaciones con parámetros ambientales y la evaluación de su estado ecológico. Libro de Resúmenes del X Congreso de la Asociación Española de Limnología y II Congreso Ibérico de Limnología. Valencia, España.

CHANEY, E., W. ELMORE \& W. S. PLATTS. 1990. Livestock grazing on western riparian areas. Washington: U.S. Environmental Protection Agency.

COMÍN, F. A. \& W. D. WILLIAMS, 1994. Parched continents: Our common future? In: Limnology now: A paradigm of planetary problems. $\mathrm{R}$. Margalef (ed.): 473-527. Elsevier, Sc. B.V., Amsterdam.

DECAMPS, H. 1996. The renewal of floodplain forests along rivers: a landscape perspective. Verh. Internat. Verein. Limnol., 26: 35-59.

D.O.C.E. 2000. Directiva 2000/60/CE del Parlamento Europeo y del Consejo de 23 de octubre de 2000 por la que se establece un marco comunitario de actuación en el ámbito de la política de aguas. D.O.C.E. L 327 de 22.12.00. 69 pp.

FAHMY, T. 1998. XLSTAT. Version 3.5.
FISHER, S. G. \& G. E LIKENS. 1973. Energy flow in Bear Brook, New Hampshire: an integrative approach to stream ecosystem metabolism. Ecol. Monogr., 43: 421-439.

GIREL, J. \& O. MANNEVILLE. 1998. Present species richness of plant communities in alpine stream corridors in relation to historical river management. Biol. Conserv., 85: 21-33.

GASITH, A. \& V.H. RESH, 1999. Streams in Mediterranean Climate Regions: Abiotic Influences and Biotic Responses to Predictable Seasonal Events. Annu. Rev. Ecol. Syst.,30: 51-81.

GONÇALVES, T. M. 2001. Centrais hidroeléctricas de pequeña dimensâo: Impacto na dinámica da comunidade de macroinvertebrados bentónicos e na variaçao da qualidade da agua (Mini-hidrica de Vila Viçosa e "Cascata do Alva"). Tesis Doctoral, Universidade do Porto, Portugal.

GONZÁLEZ-BERNÁLDEZ, F. 1988. Aspectos paisajísticos de las riberas. Centro de Estudios y Experimentación de Obras Públicas. Curso sobre Restauración de riberas modificadas por actividades de la Obras Pública. Madrid.

GONZÁLEZ DEL TÁNAGO, M., M. R. VIDALABARCA, M. L. SUÁREZ \& C. MOLINA. 1995. Estado actual de las riberas de los principales cauces fluviales de la Cuenca del Río Segura. Anales de Biología, 20 (Biología Ambiental, 9): 117-130.

GONZÁLEZ DEL TÁNAGO, M. 1999. La restauración de los ríos y el paisaje fluvial. En: Homenaje a Don Ángel Ramos Fernández (1926-1998): 641656. Real Academia de Ciencias Exactas, Físicas y Naturales, Academia de Ingeniería, Escuela Técnica Superior de Ingenieros de Montes, Madrid, España.

GONZÁLEZ DEL TÁNAGO, M. \& GARCÍA DE JALÓN, D. 1998. Restauración de Ríos y Riberas. Madrid: Mundi-Prensa. 319 pp.

GONZÁLEZ DEL TÁNAGO, M. \& N. ANTÓN. 1998. Plan forestal de la Comunidad de Madrid. Subprograma de ríos y riberas. E.T.S. Ingenieros de Montes. UPM. Madrid.

GONZÁLEZ DEL TÁNAGO, M. \& N. ANTÓN. 2000. Aplicación del índice QBR para estimar la calidad ambiental de las riberas de los principales ríos de la Comunidad de Madrid. Libro de Resúmenes del X Congreso de la Asociación Española de Limnología y II Congreso Ibérico de Limnología, Valencia, España.

GREGORY, S. V., F. J. SWANSON, W. A. MCKEE, K. \& W. CUMMINS. 1991. An ecosystem perspective of riparian zone. Bioscience, 41: 540-551. 
HAYCOCK, N., T. P. BURT, K. W. T. GOULDING \& G. PINAY. 1996. Buffer zones: Their processes and potential in water protection. Harpenden, Hertfordshire: Quest Environmental.

IBERO, C., C. ÁlvAREZ, J. C. BLANCO, J. CRIADA, A. SÁNCHEZ \& C. VIADA. 1996. Ríos de vida. El estado de conservación de las riberas fluviales en España. Sociedad Española de Ornitología, SEO/Birdlife.

JÁIMEZ-CUÉLLAR, P., S. VIVAS, N. BONADA, S. ROBLES, A. MELLADO, M. ÁlVAREZ, J. AVILÉS, J. CASAS, M. ORTEGA, I. PARDO, N. PRAT, M. RIERADEVALL, C. E. SÁINZCANTERO, A. SÁNCHEZ-ORTEGA, M. L. SUÁREZ, M. TORO, M.R. VIDAL-ABARCA, C. ZAMORA-MUÑOZ \& J. ALBA-TERCEDOR. 2002. Protocolo GUADALMED (PRECE). Limnetica, 21: 187-204.

KARR, J.R. 1999. Defining and measuring river health. Freshwat. Biol., 41: 221-234.

MARTÍNEZ, C. \& LOZANO, P. L. En prensa. Aplicación del índice de calidad del bosque de ribera, QBR (Munné et al., 1998) al Río Júcar en la Provincia de Albacete. Actas de las II Jornadas sobre el Medio Natural Albacetense, Albacete, España.

MITSCH, W. J., R. H. MITSCH \& R. E. TURNER. 1994. Wetlands of the old and new world: ecology and mangement.. In: Global wetlans: old world and new. W. J. Mitsch (ed.): 3-56. Elsevier, Amsterdam

MOLLES, M. C. JR, C. S. CRAWFORD, L. M. ELLIS, H. M. VALETT \& C.N. DAHM. 1998. Managed flooding for riparian ecosystem restoration. BioScience, 48: 749-756.

MORENO, J. L., M. L. SUÁREZ, M. R. VIDALABARCA. 1996. Valor ecológico de las ramblas como sistemas acuáticos singulares. Tomo Extraordinario 125 Aniversario de la Real Sociedad Española de Historia Natural: 411-415.

MUNNÉ A., C. SOLÁ, N. PRAT. 1998. QBR: Un índice rápido para la evaluación de la calidad de los ecosistemas de ribera. Tecnología del Agua, 175: 20-37.

MUNNÉ, A., N. PRAT, C. SOLÀ, N. BONADA \& M. RIERADEVALL. 2003. A simple field method for assessing the ecological quality of riparian habitat in rivers and streams. QBR index. Aquatic Conservation: Marine and Freshwater Ecosystems, 13: 147-164.

NAIMAN, R. J., S. R. ELLIOTT, J. M. HELFIELD \& T. C. O'KEEFE. 2000. Biophysical interactions and the structure and dynamics of riverine ecosystems: the importance of biotic feedbacks. Hydrobiologia, 410: 79-86.

OSBORNE, L. L. \& D. A. KOVACIC. 1993. Riparian vegetated buffer strips in water-quality restoration and stream management. Freshwat. Biol., 29: 243258.

PETTIT, N. E., R. H. FROEND \& P. M. DAVIES 2001. Identifying the natural flow regime and the relationship with riparian vegetation for two contrasting western Australian rivers. Regul. Rivers: Res. Mgmt., 17: 201-215.

PRAT, N. 1996. Management of freshwater resources in Mediterranean river basins. European Commission Conference on Community Water Policy. Panel 4.-Water Quantity Management. Brussels, 28-29 May 1996.

PRAT, N., M. RIERADEVALL, A. MUNNÉ, C. SOLÀ \& N. BONADA. 1999. La qualitat ecológica del Llobregat, el Besòs i el Foix. Informe 1997. Barcelona: Diputació de Barcelona. Àrea de Medi Ambient (Estudis de la Qualitat Ecológica dels Rius, 6).

PUTH L. M. \& K. A. WILSON. 2001. Boundaries and corridors as a continuum of ecological flow control: Lessons from rivers and streams. Conserv. Biol. 2001, 15: 21-30.

ROBLES, S., M. TORO, C. NUÑO, J. AVILÉS, J. ALBA-TERCEDOR, M. ÁLVAREZ, N. BONADA, J. CASAS, P. JÁIMEZ-CUÉLLAR, A. MELLADO, A. MUNNÉ, I. PARDO, N. PRAT, M. L. SUÁREZ, M. R. VIDAL-ABARCA, S. VIVAS, G. MOYÁ \& G. RAMON. 2002. Descripción de las cuencas mediterráneas seleccionadas en el proyecto GUADALMED. Limnetica, 21: 35-61.

SCHADE, J. D., S. G. FISHER, N. B. GRIMM \& J. A. SEDDON. 2001. The influence of a riparian shrub on nitrogen cycling in a Sonoran desert stream. Ecology, 82: 3363-3379.

SCHADE, J. D., E. MARTÍ, J. R. WELTER, S. G. FISHER \& N.B. GRIMM. 2002. Sources of nitrogen to the riparian zone of a desert stream: Implications for riparian vegetation and nitrogen retention. Ecosystems, 5: 68-79.

SHAFROTH, P. B., J. C. STROMBERG \& D. T. PATTEN. 2002. Riparian vegetation response to altered disturbance and stress regimes. Ecol. Appl., 12: 107-123.

SUÁREZ, M. L. \& M. R. VIDAL-ABARCA. 2000. Aplicación del índice de calidad del bosque de ribera, QBR (Munné et al., 1998) a los cauces flu- 
viales de la Cuenca del Río Segura. Tecnología del agua, 201: 33-45.

SWANSON, F. J., S. L. JOHNSON, S.V. GREGORY \& S.A. ACKER. 1998. Flood disturbance in a forested mountain landscape. BioScience, 48: 681-690.

VAN DER SMAN, A. J. M., N. N. JOOSTER \& C. W. P. M. BLOM. 1993. Flooding regimes and lifehistory characteristics of short-lived species in river forelands. J. Ecol., 81: 121-130.

VIDAL-ABARCA, M. R. 1990. Los ríos de las cuencas áridas y semiáridas: Una perspectiva ecológica comparativa y de síntesis. Scientia gerundensis, 16: 219-228.

VIDAL-ABARCA, M. R., C. MONTES, M. L. SUÁREZ \& L. RAMÍREZ-DÍAZ. 1987. El clima de la Cuenca del Río Segura: Factores que lo controlan. Anales de Biología, 12: 11-28.

VIDAL-ABARCA, M. R., M. L. SUÁREZ \& L. RAMÍREZ-DÍAZ. 1992. Ecology of Spanish semiarid streams. In: Limnology in Spain. C. Montes \& C. Duarte (eds.): 151-160. Limnetica, Asociación Española de Limnología.

VIDAL-ABARCA, M. R., M. L. SUÁREZ \& L. RAMIREZ-DÍAZ. 1996. Tipo: Ramblas/Wadis. In: Management of Mediterranean wetlands. III. Case studies 2. Proyecto MEDWET. C. Morillo \& J.L. González (eds.): 17-38. Ministerio de Medio Ambiente, Dirección General de Conservación de la Naturaleza, Unión Europea.

WARD J. V. 1998. Riverine landscapes: Biodiversity patterns, disturbance regimes, and aquatic conservation. Biol. Conserv., 83: 269-278.

ZAMORA-MUÑOZ, C., P. JÁIMEZ-CUÉLLAR \& J. ALBA-TERCEDOR. 2000. Estado ecológico de ríos mediterráneos de influencia nival del sur de la Península Ibérica. Libro de Resúmenes del X Congreso de la Asociación Española de Limnología y II Congreso Ibérico de Limnología. Valencia, España. 